\title{
Organic polaritons enable local vibrations to drive long-range energy transfer
}

\author{
R. Sáez-Blázquez, ${ }^{1}$ J. Feist, ${ }^{1}$ A. I. Fernández-Domínguez, ${ }^{1}$ and F. J. García-Vidal ${ }^{1,2, *}$ \\ ${ }^{1}$ Departamento de Física Teórica de la Materia Condensada and Condensed Matter Physics Center (IFIMAC), \\ Universidad Autónoma de Madrid, E-28049 Madrid, Spain \\ ${ }^{2}$ Donostia International Physics Center (DIPC), E-20018 Donostia/San Sebastián, Spain
}

(Received 5 April 2018; revised manuscript received 5 June 2018; published 21 June 2018)

\begin{abstract}
Long-range energy transfer in organic molecules has been experimentally obtained by strongly coupling their electronic excitations to a confined electromagnetic cavity mode. Here, we shed light into the polariton-mediated mechanism behind this process for different configurations: donor and acceptor molecules either intermixed or physically separated. We numerically address the phenomenon by means of Bloch-Redfield theory, which allows us to reproduce the effect of complex vibrational reservoirs characteristic of organic molecules. Our findings reveal the key role played by the middle polariton as the nonlocal intermediary in the transmission of excitations from donor to acceptor molecules. We also provide analytical insights on the key physical magnitudes that help to optimize the efficiency of the long-range energy transfer.
\end{abstract}

DOI: 10.1103/PhysRevB.97.241407

Energy transfer is crucial in the process of photosynthesis in biological complexes [1-3]. In recent years, fundamental research on this phenomenon has also led to the proposal of optoelectronic devices mimicking it for different functionalities [4,5]. In these systems, the exciton transmission from one organic molecule to another relies on their shortrange dipole-dipole interaction, often described by Förster theory [6]. This restricts the efficiency of the process to nanometric distances. However, by modifying the electromagnetic environment of the molecules, several experimental studies $[7,8]$ have shown that it is possible to extend the range of energy transfer to tens of nanometers.

On the other hand, very recent experimental and theoretical works have demonstrated that when a collection of organic molecules is strongly coupled to an electromagnetic mode [9-13], their chemical [14-18] and material [19-21] properties can be tailored. Regarding the energy transfer process, it has been also shown that it is feasible to enhance its efficiency by taking advantage of the phenomenon of collective strong coupling $[22,23]$. This gives rise to spatially extended hybrid light-matter states (i.e., polaritons), which can be utilized to extend the range of energy transfer to length scales comparable to the optical wavelength of the cavity mode (hundreds of nanometers) [24-27].

In this Rapid Communication, we present a theoretical description of this nonlocal energy transfer-beyond nearestneighbor interactions-taking place between two sets of organic molecules (donors and acceptors) strongly coupled to a cavity mode. The use of the Bloch-Redfield theory allows us to introduce the effect of vibrational reservoirs, which act as an effective vehicle for the excitation transmission from donor to acceptor molecules. The prevailing decay path turns out to involve the so-called middle polaritons, whose mixed

\footnotetext{
*fj.garcia@uam.es
}

composition of donor and acceptor states enables the energy transfer.

Figure 1 sketches our model system. It is composed of two collections of quantum emitters, $N_{\mathrm{D}}$ donors and $N_{\mathrm{A}}$ acceptors, between which the energy transfer is sought. Both donors and acceptors are treated as two-level systems with transition frequencies $\omega_{\mathrm{D}}$ and $\omega_{\mathrm{A}}$, respectively. They are placed, either intermixed (top panel) or separated by an intermediate wall of width $d$ (bottom panel), inside an optical cavity which supports a single mode of frequency $\omega_{\mathrm{C}}$. In the first configuration, which tries to mimic the experimental setups analyzed in Refs. [22,23], the coupling $g_{n}$ between the $n$th molecule and the electromagnetic mode is assumed to follow the spatial profile of the fundamental cavity mode. In the second scenario, $g_{n}$ presents a dependence dictated by the second cavity mode (note that the coupling strength profile exhibits a node at the position of the dividing barrier). In this way, we match the experimental configuration reported in Ref. [24]. The Hamiltonian describing these two hybrid systems within the rotating wave approximation is given by

$$
\begin{aligned}
H_{\mathrm{exc}}= & \omega_{\mathrm{C}} a^{\dagger} a+\sum_{n=1}^{N_{\mathrm{D}}} \omega_{\mathrm{D}} \sigma_{n}^{z}+\sum_{n=1}^{N_{\mathrm{A}}} \omega_{\mathrm{A}} \sigma_{n}^{z} \\
& +\sum_{n=1}^{N_{\mathrm{D}}+N_{\mathrm{A}}} g_{n}\left(a^{\dagger} \sigma_{n}+a \sigma_{n}^{\dagger}\right),
\end{aligned}
$$

where $a^{\dagger}$ and $a$ are the bosonic operators for the cavity mode, and $\sigma_{n}^{\dagger}$ and $\sigma_{n}$ are the fermionic operators for the $n$th molecule $\left(\sigma_{n}^{z} \equiv\left[\sigma_{n}^{\dagger}, \sigma_{n}\right] / 2\right)$. Notice that, within this approach, we disregard dipole-dipole coupling between molecules as its contribution to exciton transport is negligible when the polariton-mediated mechanism is fully operative [20].

Figure 2(a) shows the eigenenergies obtained from the diagonalization of $H_{\text {exc }}$ parametrized in accordance with the physical magnitudes reported in Ref. [24]. Specifically, in 


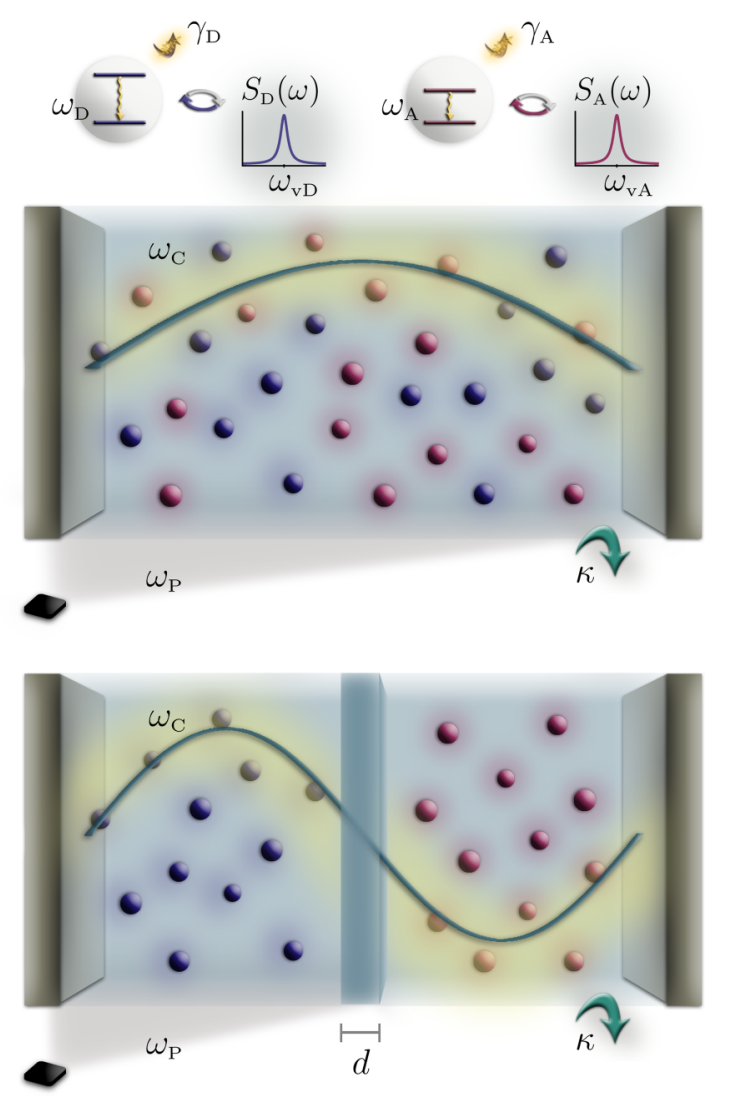

FIG. 1. Scheme of the configurations under study, where the molecules are placed in the cavity either intermixed (top) or separated by a dividing wall (bottom), with the set of parameters characterizing the model.

our study, donor and acceptor molecules are characterized by transition frequencies $\omega_{\mathrm{D}}=2.1 \mathrm{eV}$ and $\omega_{\mathrm{A}}=1.88 \mathrm{eV}$, respectively, and the cavity mode is tuned to be at resonance with $\omega_{\mathrm{D}}$. The eigenenergies are plotted as a function of the Rabi frequency, $\Omega_{\mathrm{R}} \equiv\left(\sum_{n=1}^{N_{\mathrm{D}}} g_{n}^{2}\right)^{1 / 2}$. It is important to notice that the eigenenergies only depend on $\Omega_{\mathrm{R}}$ and not on the particular set of $g_{n}$. Therefore, Fig. 2(a) is valid for both the intermixed and physically separated molecular arrangements. The energies of the upper (UP), middle (MP), and lower (LP) polaritons are depicted in blue, green, and yellow, respectively. Together with these three hybrid light-matter states, there appear $N_{\mathrm{D}}-1$ $\left(N_{\mathrm{A}}-1\right)$ dark superpositions of donor (acceptor) states. These constitute the $\mathcal{D}$ and $\mathcal{A}$ subspaces, degenerate at the energies of the bare donor (gray dashed-dotted line) and acceptor (gray dashed line) bare molecules, respectively.

The cavity and matter components of each polariton are plotted in Fig. 2 as a function of the Rabi splitting [Fig. 2(b)] and the cavity frequency [Fig. 2(c)]. The Hopfield coefficients $b_{\alpha \iota}$ (where $\iota=\mathrm{C}, \mathrm{D}$, or A) describe the contents of the cavity (C), donor (D), and acceptor (A) in the state $\alpha$ of the polariton basis (UP, MP, and LP). For the set of parameters considered, the UP branch mainly results from the hybridization of the cavity and donor molecules, while the LP branch is composed mostly of acceptor states mixed with the optical field. This is why UP and LP are essentially identified with donor and acceptor molecules, respectively, although the presence of

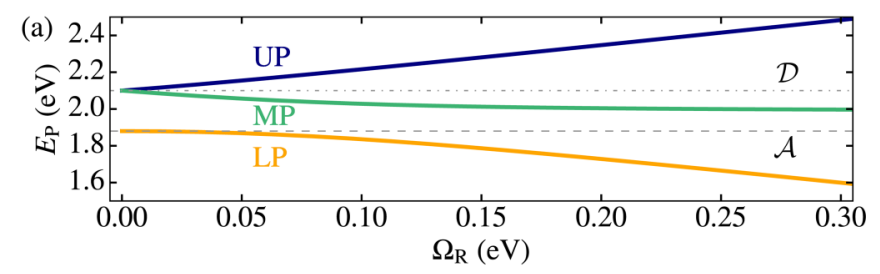

(b)
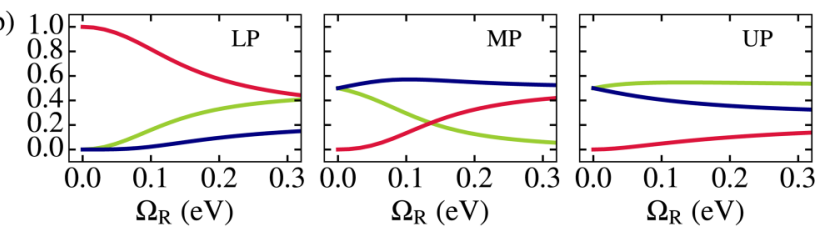

(c)
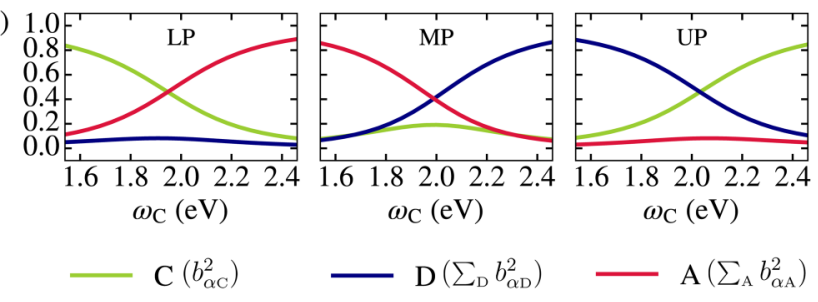

$\omega_{\mathrm{C}}(\mathrm{eV})$

$\mathrm{A}\left(\sum_{\mathrm{A}} b_{\alpha \mathrm{A}}^{2}\right)$

FIG. 2. (a) Energies of the UP (blue), MP (green), and LP (yellow) as a function of the Rabi frequency $\Omega_{R}$. Dashed and dashed-dotted lines stand for the donor and acceptor energies and their associated dark states $\mathcal{D}$ and $\mathcal{A}$. (b), (c) Coefficients representing the cavity (green), donor (blue), and acceptor (red) components of the LP (left), MP (center), and UP (right) as a function of the Rabi frequency $\Omega_{R}$ for $\omega_{\mathrm{C}}=2.1 \mathrm{eV}$ in (b), and the cavity frequency $\omega_{\mathrm{C}}$ with $\Omega_{\mathrm{R}}=0.16 \mathrm{eV}$ in panels (c).

other components should not be overlooked. On the contrary, the MP branch contains a mixture of states in which both types of molecules have similar weights, which has profound implications for long-range energy transfer.

In order to study polariton-mediated energy transfer in the systems depicted in Fig. 1, our description must go beyond Eq. (1). First, we need to add a new term in the Hamiltonian $H_{\mathrm{P}}=\Omega_{\mathrm{P}}\left(a^{\dagger} e^{-i \omega_{\mathrm{P}} t}+a e^{i \omega_{\mathrm{P}} t}\right)$, accounting for the coherent pumping of the optical cavity by a laser of frequency $\omega_{\mathrm{P}}$ and driving strength $\Omega_{\mathrm{P}}$. Within an open quantum system treatment based on solving the master equation for the density matrix, we describe the dissipation experienced by molecular excitations due to their internal vibronic structure by means of the general Bloch-Redfield approach [28-30]. This requires the inclusion of spectral densities $S_{n}(\omega)$ characterizing the local vibrational reservoir of each molecule $n$. Finally, radiative losses associated with donors and acceptors (with decay rates $\gamma_{\mathrm{D}}$ and $\gamma_{\mathrm{A}}$, respectively), as well as cavity losses (with decay rate $\kappa$ ), are modeled by means of Lindblad superoperators [31,32].

We consider a simplified system in our numerical simulations. It consists of $N_{\mathrm{D}}=16$ donors and $N_{\mathrm{A}}=16$ acceptors extended over a length of $100 \mathrm{~nm}$ and separated by a wall of $d=10 \mathrm{~nm}$. The molecules are coupled to a cavity mode with a Rabi splitting $\Omega_{\mathrm{R}}=0.16 \mathrm{eV}$, with $g_{n}$ following the spatial profile of the second cavity mode. The (nonradiative and radiative) losses of the cavity are set to $\kappa=0.01 \mathrm{eV}$, and the radiative losses of the molecules are $\gamma_{\mathrm{D}}, \gamma_{\mathrm{A}}=1.3 \mu \mathrm{eV}$, which are typical values for organic molecules. The laser is 
(a)

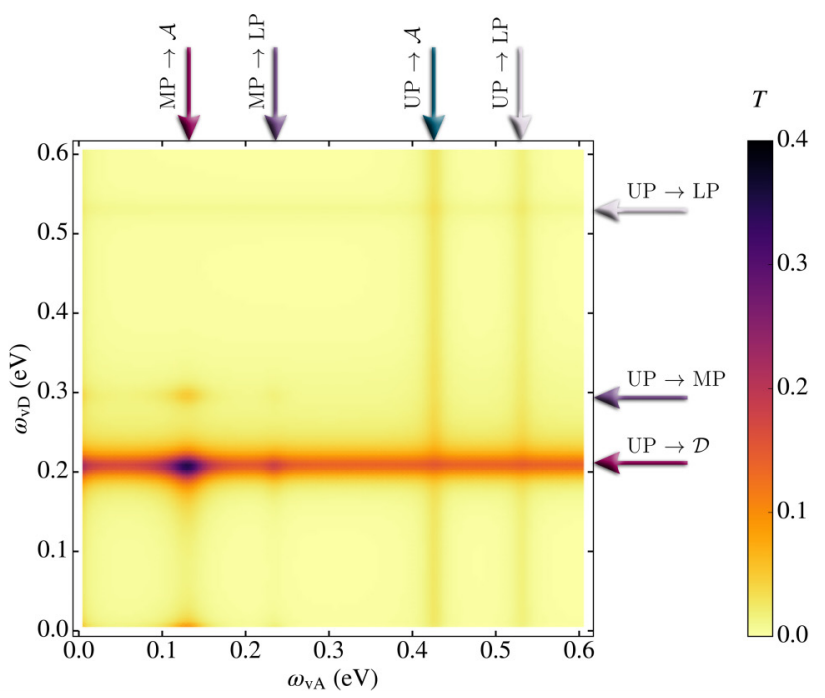

(b)

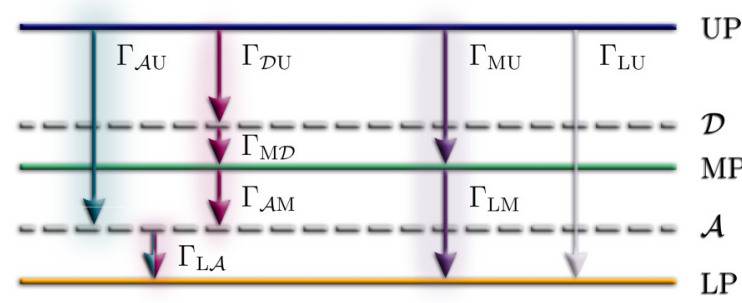

$\vdots$

FIG. 3. (a) Transfer efficiency $T$ for $N_{\mathrm{D}}=16$ donors and $N_{\mathrm{A}}=16$ acceptors, coupled such that the Rabi splitting is $\Omega_{\mathrm{R}}=0.16 \mathrm{eV}$, when varying the center frequencies $\omega_{\mathrm{vA}}$ and $\omega_{\mathrm{vD}}$ of the Lorentzians representing the thermal bath associated with donor and acceptor molecules, respectively. The corresponding transitions are marked by arrows on the top and right edges. (b) Level scheme of the polaritons (as well as the two sets of dark states $\mathcal{D}$ and $\mathcal{A}$ ) showing various paths of decay, which are labeled with their corresponding rates $\Gamma$.

tuned to pump the cavity at the UP frequency, so that all the excitation is placed in this polariton and, consequently, mainly in the donor molecules. To investigate the role of vibrations in the UP-to-LP transfer of excitations, we fix all the parameters of the model except for the spectral densities mimicking the molecular vibrational reservoirs. Two Lorentzian-like profiles vanishing at zero frequency are chosen to describe them,

$$
S_{l}(\omega)=\gamma_{\phi} \frac{\omega}{\omega_{\mathrm{v} l}} \frac{\xi^{2}}{\left(\omega-\omega_{\mathrm{v} l}\right)^{2}+\xi^{2}},
$$

where $\omega_{\mathrm{v} \iota}$ are the resonant vibrational frequencies $(\iota=\mathrm{D}, \mathrm{A}$ stand for donors and acceptors, respectively). The strength of the exciton-phonon coupling is parametrized by $\gamma_{\phi}$, which determines the phonon-induced transition rate on resonance. In our calculations we have used $\gamma_{\phi}=0.013 \mathrm{eV}$ that corresponds to the relatively small exciton-phonon coupling present in $J$-aggregates, hence it would be greater if considering monomeric chromophores that present larger Stokes shift values. Regarding the linewidth of these vibrational modes, we consider a large value, $\xi=0.01 \mathrm{eV}$, in order to account for the effective broadening of the resonances due to cavity losses $(\xi \approx \kappa)$.

We theoretically quantify the energy transfer process in a similar way to experiments, where the light emission at the LP is measured, by defining the transfer efficiency $T$ as

$$
T=\frac{b_{\mathrm{LC}}^{2} P_{\mathrm{L}}}{b_{\mathrm{LC}}^{2} P_{\mathrm{L}}+b_{\mathrm{MC}}^{2} P_{\mathrm{M}}+b_{\mathrm{UC}}^{2} P_{\mathrm{U}}},
$$

where $P_{\alpha}$ is the population of polariton $\alpha$, whose cavity content is given by coefficient $b_{\alpha \mathrm{C}}$. Note that $T$ gives the contrast between the emission from the LP and the total light leaking from the cavity under coherent pumping. Figure 3(a) renders $T$ as a function the two resonant vibrational frequencies, $\omega_{\mathrm{vD}}$ and $\omega_{\mathrm{vA}}$. The relevant energy differences between eigenstates are indicated in the margins. The contour plot reveals the conditions for which $T$ is enhanced. We can observe first a vertical line, which originates from the resonant tuning of $S_{\mathrm{A}}(\omega)$ with the transition from the UP to the $\mathcal{A}$ states. This feature cannot be attributed to an energy transfer process, since it is the acceptor component of the UP that directly produces it. This enhancement of exciton transport mediated by polaritons has already been predicted $[20,33]$. There are also two equivalent high- $T$ lines, one horizontal and one vertical, where the vibronic frequencies are the same as the energy gap between the UP and the LP. Apart from those, a more pronounced enhancement of the transfer efficiency takes place when $S_{\mathrm{D}}(\omega)$ peaks at the energy gap between the UP and the $\mathcal{D}$ states. Importantly, $T$ is maximum at a single point along this horizontal line. This maximum emerges when $\omega_{\mathrm{vA}}$ also matches the energy difference between the MP and the $\mathcal{A}$ states.

Therefore, our numerical results reveal that the main pathway that leads to energy transfer from donor to acceptor molecules carries the population through the MP owing to local exciton-vibration interactions. The mixed composition of the MP, which combines donor and acceptor molecule populations in similar proportions, boosts the population transfer. This prevailing path corresponds to the red route depicted in Fig. 3(b), where the other possible processes are also shown: the direct decay of the UP into $\mathcal{A}$ states (green) and the channels involving only polaritons (purple and gray). Notice that this boost in the energy transfer requires both donor and acceptor molecules to be strongly coupled to the cavity mode, and the achievable enhancement is strongly reduced when only one of the collections becomes hybridized [26].

Importantly, we have verified that the general picture offered by Fig. 3 on the link between energy transfer and molecular vibrations is not altered, even at the quantitative level, when donor and acceptor molecules are intermixed, as long as the Rabi splitting remains the same. This demonstrates that $\Omega_{\mathrm{R}}$ is the only key parameter describing the effect of light-matter coupling in the process of energy transfer, which is independent 
of aspects such as the actual molecular arrangement or the spatial dependence of the cavity mode, in agreement with experimental results [24].

Our numerical analysis also shows that, for the steady-state solution, terms coupling the off-diagonal (coherences) and diagonal (populations) elements of the density matrix can be disregarded in the Bloch-Redfield master equation. Under this approximation (equivalent to the secular approximation), we can now restrict our attention to just the transition rates connecting polaritonic and/or dark states, as depicted in Fig. 3(b). This greatly simplifies the numerical treatment, making the theoretical study of systems involving a much larger number of donor and acceptor molecules feasible. Moreover, as shown below, we can obtain analytical expressions for the relevant decay rates, expressed only in terms of the Hopfield coefficients and the vibronic spectral densities.

Depending on the nature of the states involved in the transition, we can identify three different sets of decay rates. First, those three connecting two polariton states $\alpha$ and $\beta$ can be expressed as

$$
\Gamma_{\alpha \beta}=\sum_{\iota=\mathrm{D}, \mathrm{A}} b_{\beta l}^{2} b_{\alpha \iota}^{2} S_{\iota}\left(\omega_{\beta}-\omega_{\alpha}\right)
$$

where the dependence of $b_{\alpha \iota}$ on the number of molecules is of the form $b_{\alpha \iota} \sim 1 / \sqrt{N_{\iota}}$, yielding $\Gamma_{\alpha \beta} \sim 1 / N_{\mathrm{A}, \mathrm{D}}$. Equation (4) reveals that $\Gamma_{\mathrm{MU}}, \Gamma_{\mathrm{LM}}$, and $\Gamma_{\mathrm{LU}}$ vanish as the number of molecules increases, and the contributions to the energy transfer from the purple and gray routes in Fig. 3(b) are negligible in very large systems.

Second, the rates for the decay of the dark subspaces to a polariton state of lower energy are given by

$$
\Gamma_{\alpha \iota^{\prime}}=\frac{1}{N_{\iota}} \sum_{\iota} b_{\alpha \iota}^{2} S_{\iota}\left(\omega_{\iota}-\omega_{\alpha}\right)
$$

where $\iota=\mathrm{D}(\iota=\mathrm{A})$ for $\iota^{\prime}=\mathcal{D}\left(\iota^{\prime}=\mathcal{A}\right)$ and $\alpha$ denotes LP for $\iota^{\prime}=\mathcal{A}$ and LP or MP when $\iota^{\prime}=\mathcal{D}$. Equation (5) indicates that $\Gamma_{\mathrm{M} \mathcal{D}}, \Gamma_{\mathrm{L} \mathcal{D}}$, and $\Gamma_{\mathrm{L} \mathcal{A}}$ present the same $1 / N_{\mathrm{A}, \mathrm{D}}$ dependence as the decay rates between polaritonic states. However, in contrast to decay from polariton states (which decay efficiently by cavity leakage of their photonic contribution), the competing decay paths due to bare-molecule radiative and nonradiative decay are typically on the order of nanoseconds for high-quantum-yield emitters. Consequently, even slow decay from the dark states efficiently populates the lower-lying polaritons.

Finally, the transition rates from polariton states to dark subspaces that have lower energies have the form

$$
\Gamma_{\iota^{\prime} \alpha}=\frac{N_{\iota}-1}{N_{\iota}} \sum_{\iota} b_{\alpha \iota}^{2} S_{\iota}\left(\omega_{\alpha}-\omega_{\iota}\right),
$$

where the $N_{\iota}-1$ term reflects the (large) number of dark states to which polaritons can decay. Equation (6) yields $\Gamma_{\mathcal{D U}} \sim\left(N_{\mathrm{D}}-1\right) / N_{\mathrm{D}}$ and $\Gamma_{\mathcal{A U}}, \Gamma_{\mathcal{A M}} \sim\left(N_{\mathrm{A}}-1\right) / N_{\mathrm{A}}$. Thus, these three decay rates do not decrease as the number of molecules increases, as the other six rates do. This gives analytical support to our Bloch-Redfield numerical results that showed the prevalence of the red route in the decay of the excitation from the UP to the LP.

Our analytical results not only explain the numerical findings previously discussed but also serve as a guideline to optimize the long-range energy transfer mediated by strong coupling. In order to enhance the transfer efficiency, the vibration-driven decay $\Gamma_{\mathcal{D U}}\left(\Gamma_{\mathcal{A M}}\right)$ from the UP $(\mathrm{MP})$ polariton to the $\mathcal{D}(\mathcal{A})$ dark states has to be comparable to or faster than its decay $b_{\mathrm{UC}}^{2} \kappa\left(b_{\mathrm{MC}}^{2} \kappa\right)$ due to cavity losses. This can be achieved most straightforwardly by using cavities with very low losses. However, for the given cavity losses, optimization relies on minimizing the cavity component of the upper and middle polaritons while maximizing the donor (acceptor) component of UP (MP). Both these conditions favor low cavity frequencies [see Fig. 2(c)]. In addition, the vibration-driven decay can be enhanced by bringing the energy detuning between UP and $\mathcal{D}$ $(\mathrm{MP}$ and $\mathcal{A}$ ) into resonance with the main vibronic frequency of the donor (acceptor) molecules. Another way to enhance both $\Gamma_{\mathcal{D U}}$ and $\Gamma_{\mathcal{A M}}$ is to employ organic molecules with a large $\gamma_{\phi}$, as these decay rates are proportional to the spectral densities [see Eqs. (6) and (2)]. Our model envisages that transfer efficiencies close to $100 \%$ can be reached under the conditions $\Gamma_{\mathcal{D U}} \gg b_{\mathrm{UC}}^{2} \kappa$ and $\Gamma_{\mathcal{A M}} \gg b_{\mathrm{MC}}^{2} \kappa$.

To conclude, we have presented both a numerical treatment based on the Bloch-Redfield formalism and an analytical approach to underpin the physics of the phenomenon of longrange energy transfer mediated by collective strong coupling. We have demonstrated the key role played by the delocalized character of the middle polariton in this process as it enables the vibrations to transfer the excitation from donor to acceptor molecules. Importantly, this nonlocal energy transfer is dominated by the Rabi frequency and does not depend on the particular arrangement of the molecules inside the cavity or the electromagnetic mode spatial profile. Therefore, as long as collective strong coupling is achieved, our theoretical results predict that there is no limit in the physical separation attainable between donor and acceptor molecules. Not only have we been able to unveil the physical mechanism behind vibration-driven long-range energy transfer, but our analytical approach has allowed us to deliver specific recipes to optimize the phenomenon.

We thank J. del Pino for fruitful discussions. This work has been funded by the European Research Council under Grant Agreements No. ERC-2011-AdG 290981 and No. ERC2016-STG-714870, the EU Seventh Framework Programme (FP7-PEOPLE-2013-CIG-630996 and FP7-PEOPLE-2013CIG-618229), and the Spanish MINECO under Contracts No. MAT2014-53432-C5-5-R and No. FIS2015-64951-R, as well as through the "María de Maeztu" programme for Units of Excellence in R\&D (MDM-2014-0377).
[1] R. van Grondelle and V. I. Novoderezhkin, Phys. Chem. Chem. Phys. 8, 793 (2006).
[2] G. D. Scholes, G. R. Fleming, A. Olaya-Castro, and R. van Grondelle, Nat. Chem. 3, 763 (2011). 
[3] A. Olaya-Castro and G. D. Scholes, Int. Rev. Phys. Chem. 30, 49 (2011).

[4] M. A. Baldo, M. E. Thompson, and S. R. Forrest, Nature (London) 403, 750 (2000).

[5] B. E. Hardin, E. T. Hoke, P. B. Armstrong, J.-H. Yum, P. Comte, T. Torres, J. M. Frechet, M. K. Nazeeruddin, M. Gratzel, and M. D. McGehee, Nat. Photon. 3, 406 (2009).

[6] T. Förster, Ann. Phys. 437, 55 (1948).

[7] P. Andrew and W. L. Barnes, Science 306, 1002 (2004).

[8] S. Gotzinger, L. de S. Menezes, A. Mazzei, S. Kuhn, V. Sandoghdar, and O. Benson, Nano Lett. 6, 1151 (2006).

[9] I. Pockrand, A. Brillante, and D. Mobius, J. Chem. Phys. 77, 6289 (1982).

[10] D. G. Lidzey, D. D. C. Bradley, M. S. Skolnick, T. Virgili, S. Walker, and D. M. Whittaker, Nature (London) 395, 53 (1998).

[11] J. Bellessa, C. Bonnand, J. C. Plenet, and J. Mugnier, Phys. Rev. Lett. 93, 036404 (2004).

[12] J. Dintinger, S. Klein, F. Bustos, W. L. Barnes, and T. W. Ebbesen, Phys. Rev. B 71, 035424 (2005).

[13] P. Törmä and W. L. Barnes, Rep. Prog. Phys. 78, 013901 (2015).

[14] J. A. Hutchison, T. Schwartz, C. Genet, E. Devaux, and T. W. Ebbesen, Angew. Chem. 124, 1624 (2012).

[15] J. Galego, F. J. Garcia-Vidal, and J. Feist, Phys. Rev. X 5, 041022 (2015).

[16] F. Herrera and F. C. Spano, Phys. Rev. Lett. 116, 238301 (2016).

[17] K. Bennett, M. Kowalewski, and S. Mukamel, Faraday Discuss. 194, 259 (2016).

[18] J. Flick, C. Schäfer, M. Ruggenthaler, H. Appel, and A. Rubio, ACS Photon. 5, 992 (2018).
[19] E. Orgiu, J. George, J. A. Hutchison, E. Devaux, J. F. Dayen, B. Doudin, F. Stellacci, C. Genet, J. Schachenmayer, C. Genes, G. Pupillo, P. Samorì, and T. W. Ebbesen, Nat. Mater. 14, 1123 (2015).

[20] J. Feist and F. J. Garcia-Vidal, Phys. Rev. Lett. 114, 196402 (2015).

[21] J. Schachenmayer, C. Genes, E. Tignone, and G. Pupillo, Phys. Rev. Lett. 114, 196403 (2015).

[22] D. M. Coles, N. Somaschi, P. Michetti, C. Clark, P. G. Lagoudakis, P. G. Savvidis, and D. G. Lidzey, Nat. Mater. 13, 712 (2014).

[23] X. Zhong, T. Chervy, S. Wang, J. George, A. Thomas, J. A. Hutchison, E. Devaux, C. Genet, and T. W. Ebbesen, Angew. Chem. Int. Ed. 55, 6202 (2016).

[24] X. Zhong, T. Chervy, L. Zhang, A. Thomas, J. George, C. Genet, J. A. Hutchison, and T. W. Ebbesen, Angew. Chem. Int. Ed. 56, 9034 (2017).

[25] F. J. Garcia-Vidal and J. Feist, Science 357, 1357 (2017).

[26] M. Du, L. A. Martinez-Martinez, R. F. Ribeiro, Z. Hu, V. M. Menon, and J. Yuen-Zhou, arXiv:1711.11576.

[27] M. Reitz, F. Mineo, and C. Genes, Sci. Rep. 8, 9050 (2018).

[28] F. Bloch, Phys. Rev. 105, 1206 (1957).

[29] A. G. Redfield, IBM J. Res. Dev. 1, 19 (1957).

[30] R. K. Wangsness and F. Bloch, Phys. Rev. 89, 728 (1953).

[31] H. Breuer and F. Petruccione, The Theory of Open Quantum Systems (Oxford University Press, Oxford, UK, 2002).

[32] H. J. Carmichael, Statistical Methods in Quantum Optics 1 (Springer, Berlin, 1999).

[33] C. Gonzalez-Ballestero, J. Feist, E. Moreno, and F. J. GarciaVidal, Phys. Rev. B 92, 121402 (2015). 